\title{
Identification of new sources of resistance to stem rust race Ug99 (TTKSK) in wheat genotypes
}

\author{
Fentaw Abate ${ }^{1 *}$, Patrick Rubaihayo ${ }^{1}$, Thomas Odong ${ }^{1}$, Seid Ahmed ${ }^{2}$ and Hailay Mehari ${ }^{1}$ \\ 1Department of Agricultural production, school of Agriculture, college of Agricultural and environmental sciences, \\ Makerere University, Kampala, Uganda. \\ 2International Center for Agricultural Research in the Dry Areas (ICARDA), Rabat-Institutes, Rabat, Morocco. \\ *Corresponding author. Email: fenti2004@gmail.com
}

Copyright (c) 2019 Abate et al. This article remains permanently open access under the terms of the Creative Commons Attribution License 4.0, which permits unrestricted use, distribution, and reproduction in any medium, provided the original work is properly cited.

Received 26th November, 2019; Accepted 30th December, 2019

\begin{abstract}
Stem rust disease which caused by Puccinia graminis f. sp. tritici is one of the major wheat production constraints in the high lands of central, south eastern and north western part of Ethiopia. The disease had caused up to $100 \%$ yield loss on the unprotected wheat farms. This study was conducted to identify new sources of resistance to stem rust disease. After the removal of genotypes that showed a major gene (race specific) resistance expression at seedling test, 60 selected genotypes were evaluated for their adult plant resistance to stem rust and agronomic traits across three locations (Debrezeit, Adet and Kulmsa) using a $5 \times 12$ alpha lattice design with three replications. Genotypes showed a highly significant difference $(p=0.001)$ on the traits of final rust severity (FRS), area under disease progress curve (AUDPC), coefficient of infection (Cl) and apparent infection rate $(r)$ at different locations. Moreover, combined analysis of variance also showed the presence of highly significant effects of genotypes, environments and GE interaction on the magnitude of FRS, AUDPC, $\mathrm{Cl}, \mathrm{r}$, days to maturity (DM), plant height $(\mathrm{PH})$, hectoliter weight $(\mathrm{HLW})$, thousands kernel weigh (TKW) and grain yield (GY) across testing locations. The correlation analysis revealed the presence of positive highly significant $(p<0.001)$ relationship of FRS with both $\mathrm{Cl}(r=0.894)$ and AUDPC $(r=0.877)$. A positive and highly significant $(p<0.001)$ correlation was also observed between $\mathrm{Cl}$ and AUDPC $(r=0.996)$ of the tested genotypes. Principal component analysis indicated that only the first four principal components (PCs) explained $86.58 \%$ of the total variation among the tested genotypes. Cluster analysis also confirmed the presence of variation among the tested genotypes by dividing them into five major groups. In this study, four bread wheat genotypes (G12, G60, G31 and G52) were found to be resistant to stem rust disease across the three locations and could be used as source of stem rust resistance in future wheat improvement program.
\end{abstract}

Keywords: AUDPC, coefficient of infection rate, cluster analysis, final rust severity, stem rust.

\section{INTRODUCTION}

Wheat stem rust disease which caused by the fungus Puccinia graminis $\mathrm{f}$. $\mathrm{sp}$. Tritici was first reported in Uganda in 1999 and has now spread to different wheat producing countries of the world (Singh et al., 2011). The disease appeared in Ethiopia in 2003 and became a major production constraint in wheat-growing areas of the country (Priyamvada et al., 2011). It has the potential of causing up to $100 \%$ yield losses in the unprotected wheat fields (Hailu et al., 2015). Tremendous efforts were done to control this devastating disease by spraying fungicides, though the method was not sustainable to small scale farmers due to the unaffordable chemical costs and unfriendly consequence of fungicides on the health of human being and their surrounding environments (Jaleta et al., 2019). Host plant resistance is the most economical feasible and ecologically safe method for controlling wheat stem rust disease (Gamalat and El-sawi, 2015). Accordingly, efforts have been put in looking for novel 
sources of resistance to this destructive disease. There is, however, a potential of resistance breakdown among the deployed resistant wheat varieties as there is frequent appearance of new virulent pathotypes of Ug99 and its highly adapted long distance migration of uredospores through wind and rain deposition (Singh et al., 2011).

In Ethiopia, resistance breeding for wheat rust disease was conducted by various national and regional agricultural research centers and developed many improved varieties which were resistant to stem rust disease. While after the appearance of race Ug99 in Ethiopia, most of the improved wheat varieties such as 'Lacketch', 'Kubsa' and 'Enkoy' were removed from production (Beteselassie et al., 2007) and the national average productivity of wheat declined from $2.1 \mathrm{t} / \mathrm{ha}$ to $1.83 \mathrm{t} / \mathrm{ha}$, which was $45 \%$ lower than the average wheat productivity of the world (Sahoo et al., 2016). Hence, in order to improve the national average wheat productivity, it was vital that undergoing extensive screening of various wheat genotypes to explore novel sources of resistance genes to Ug99 and incorporate those effective genes into new high yield commercial varieties. Thus, in this research, various bread wheat genotypes which were collected from International Centre for Maize and Wheat improvement (CIMMYT), International Centre for Agricultural Research in the Dry Areas (ICARDA), Debrezeit, Kulmsa and Adet Agricultural Research Centers were evaluated against stem rust disease that caused by race Ug99.

\section{MATERIALS AND METHODS}

The experiment was conducted across three stem rust hot spot locations: Debrezeit, Adet and Kulumsa Agricultural Research Centers in the main growing season of year 2016. Detail description of experimental sites is indicated in Table 1.

\section{Experimental materials}

A total of 120 bread wheat genotypes: 85 from International Centre for Maize and Wheat improvement (CIMMYT), 20 from International Centre for Agricultural Research in the Dry Areas (ICARDA) and 15 promising genotypes from Debrezeit, Kulmsa and Adet Agricultural Research Centers along with susceptible check (PBW343) were used for seedling resistance test against race Ug99 under controlled greenhouse conditions. After the removal of genotypes that showed a major gene resistance expression at seedlings resistance test, a panel of 60 wheat genotypes which exhibited mixed (intermediate and susceptible) and susceptible infection types were used to conduct adult plant resistance test against stem rust (puccinia graminis) disease at Debrezeit, Adet and Kulmsa Agricultural Research Centers which are internationally known as stem rust disease hot spot area. The description of the tested genotypes is presented in Table 2.

\section{Experimental design and management}

\section{Seedling resistance test}

Evaluation of 120 bread wheat genotypes against race TTKSK (Ug99) was carried out under controlled greenhouse conditions at their seedling stage. Five seedlings were grown per genotype in each $10 \mathrm{~cm}$ diameter plastic pot which filled with compost, light soil and sand at a $1: 1: 1$ ratio $(\mathrm{v} / \mathrm{v} / \mathrm{v})$ respectively. Until the seedlings were ready for inoculation, they were kept in microclimate room that had a temperature of 15 to $20^{\circ} \mathrm{C}$. Before inoculation, urediniospores of Pgt race TTKSK which maintained at liquid nitrogen tank were heat-shocked for 10 minutes in a water bath at $40^{\circ} \mathrm{C}$ and kept in a rehydration chamber maintained in a $\mathrm{KOH}$ solution for 2 to 4 hours with $80 \%$ relative humidity (Jin et al., 2007). Then spores were suspended in a light mineral oil, Soltrol 170 light oil (Chevron Phillips Chemical Company, The Woodlands, TX) and inoculation of seedlings with spore suspension adjusted to $4 \times 10^{6}$ spores $\mathrm{ml}^{-1}$ was conducted using spore inoculators when seedlings were reached at 2 to 3 leaf stage. Subsequently, the inoculated seedlings were placed in a dew chamber in darkness for 18 hours at 18 to $22^{\circ} \mathrm{C}$ and 98 to $100 \%$ relative humidity. Then inoculated plants were transferred to glass compartments in greenhouse where the temperature and the relative humidity were kept in with a range of 18 to $25^{\circ} \mathrm{C}$ and 60 to $70 \%$ respectively, for 12 hours photoperiod (Stubbs et al., 1986).

\section{Adult plant resistance test}

It was conducted using alpha lattice design $(5 \times 12)$ with three replications. Each genotype was planted on two adjacent rows that had $1 \mathrm{~m}$ row length with inter-row spacing of $20 \mathrm{~cm}$. The well-known most susceptible bread wheat variety (Morocco) was planted as spreader plant around the four sides of experimental area while variety PBW343 was used as susceptible check. To ensure uniform disease pressure and inoculums dissemination, seven-day-old seedlings of spreader plants were inoculated with the water-spores mixture (approximately 3 to $5 \mathrm{mg}$ of freshly collected spores per $1 \mathrm{ml}$ of distilled water suspension) and then the spreader plants were sprayed with urediniospores suspended in light mineral oil Soltrol 170 (Chevron Phillips Chemical Company, The Woodlands, TX) (Sikharulidze et al., 2015). The recommended fertilizer rate $(50 \mathrm{~kg} / \mathrm{ha}$ urea and $100 \mathrm{~kg} / \mathrm{ha}$ DAP) and seed rate $(150 \mathrm{~kg} / \mathrm{ha})$ were used in the experiment (Haile et al., 2012). The whole amount of DAP 
Table 1. Description of study areas.

\begin{tabular}{lccccccc}
\hline \multirow{2}{*}{ Locations } & $\begin{array}{c}\text { Altitude } \\
(\mathbf{m a s})\end{array}$ & Rainfall & Soil type & \multicolumn{2}{c}{ Global Position } & \multicolumn{2}{c}{ Temperature $\left({ }^{\circ} \mathbf{C}\right)$} \\
\cline { 5 - 8 } & $\mathbf{( m m )}$ & & Latitude & Longitude & Min & Max \\
Debrezeit & 1900 & 851 & clay loam & $8^{\circ} 44^{\prime} \mathrm{N}$ & $38^{\circ} 58^{\prime} \mathrm{E}$ & 8.9 & 28.3 \\
Adet & 2216 & 1331 & Nitosol & $11^{\circ} 16^{\prime} \mathrm{N}$ & $37^{\circ} 29^{\prime} \mathrm{E}$ & 9.2 & 25.5 \\
Kulmsa & 2210 & 832 & clay loam & $8^{\circ} 00^{\prime} \mathrm{N}$ & $3^{\circ} 07^{\prime} \mathrm{E}$ & 9.9 & 23.1 \\
\hline
\end{tabular}

Sources: (Denbel et al., 2013; Tamene et al., 2015; Zemede et al., 2019).

Table 2. Description of the tested bread wheat genotypes.

\begin{tabular}{|c|c|c|}
\hline Geno & Code & Pedigree \\
\hline G1 & 6003 & KINGBIRD \#1 \\
\hline \multirow{2}{*}{ G2 } & \multirow{2}{*}{6006} & MUTUS/DANPHE \#1/4/C80.1/3*BATAVIA//2*WBLL1/3/C80.1/3* \\
\hline & & QT4522//2*PASTOR \\
\hline G3 & 6008 & BAJ \#1/5/ATTILA/3*BCN//BAV92/3/TILHI/4/SHA7/VEE\#5//ARIV92 \\
\hline G4 & 56016 & PAURAQ/4/MARCHOUCH*4/SAADA/3/2*FRET2/KUKUNA//FRET2 \\
\hline G5 & 41441 & unknown \\
\hline \multirow{2}{*}{ G6 } & \multirow{2}{*}{6019} & INQALAB 91*2/KUKUNA//PFAU/WEAVER/3/INQALAB 91*2/KUK \\
\hline & & UNA/4/TRCH/SRTU//KACHU \\
\hline \multirow{2}{*}{ G7 } & \multirow{2}{*}{6020} & INQALAB 91*2/KUKUNA//PFAU/WEAVER/3/INQALAB 91*2/KUK \\
\hline & & UNA/4/TRCH/SRTU//KACHU \\
\hline \multirow{2}{*}{ G8 } & \multirow{2}{*}{6021} & INQALAB 91*2/KUKUNA//PFAU/WEAVER/3/INQALAB 91*2/KUK \\
\hline & & UNA/4/TRCH/SRTU//KACHU \\
\hline G9 & 6023 & WHEAR/SOKOLL/3/TRCH/SRTU//KACHU \\
\hline G10 & 6024 & SW2148/2*ROLF07/3/HUW234+LR34/PRINIA*2//SNLG \\
\hline G11 & KAKABA & Unknown \\
\hline \multirow{2}{*}{ G12 } & \multirow{2}{*}{6027} & WAXWING/7/TNMU/6/CEP80111/CEP81165/5/IAC5/4/YKT406/3/AG/ \\
\hline & & ASN//ATR/8/ATTILA/3*BCN//BAV92/3/TILHI/4/ SHA7/VEE\#5//ARIV92 \\
\hline G13 & 127812 & unknown \\
\hline \multirow{2}{*}{ G14 } & \multirow{2}{*}{6028} & WAXWING/7/TNMU/6/CEP80111/CEP81165/5/IAC5/4/YKT406/3/AG/ \\
\hline & & ASN//ATR/8/ATTILA/3*BCN//BAV92/3/TILHI/4/SHA7/VEE\#5//ARIV92 \\
\hline G15 & 6036 & $\mathrm{KACHU/SAUAL/3/TRCH/SRTU//KACHU}$ \\
\hline \multirow{2}{*}{ G16 } & \multirow{2}{*}{6049} & KACHU/6/WHEAR/4/SNI/TRAP\#1/3/KAUZ*2/TRAP//KAUZ/5/C80.1/ \\
\hline & & 3*BATAVIA//2*WBLL1/7/TRCH/SRTU//KACHU \\
\hline G17 & 6062 & ATTILA*2/PBW65//KIRITATI/3/QUELEA \\
\hline \multirow{2}{*}{ G18 } & \multirow{2}{*}{6066} & PF74354//LD/ALD/4/2*BR12*2/3/JUP//PAR214*6/FB6631/5/NL750/ \\
\hline & & 6/PVN/7/TOBA97/PASTOR/8/UP2338*2/KKTS*2// YANAC \\
\hline \multirow{2}{*}{ G19 } & \multirow{2}{*}{6081} & FRANCOLIN \#1/3/PBW343*2/KUKUNA*2//YANAC/4/KINGBIRD \# \\
\hline & & $1 / /$ INQALAB 91*2/TUKURU \\
\hline G20 & 6086 & MELON//FILIN/MILAN/3/FILIN/4/2*TRCH/SRTU//KACHU \\
\hline \multirow{2}{*}{ G21 } & \multirow{2}{*}{6091} & ATTILA*2/PBW65//TAM200/TUI/5/2*SERI.1B//KAUZ/HEVO/3/ \\
\hline & & AMAD*2/4/KIRITATI \\
\hline \multirow{2}{*}{ G22 } & \multirow{2}{*}{6095} & ATTILA*2/PBW65/5/CNO79//PF70354/MUS/3/PASTOR/4/BAV92/6/KI \\
\hline & & NGBIRD\#1/7/CNO79//PF70354/MUS/3/PASTOR/4/BAV92*2/5/FH6-1-7 \\
\hline \multirow{2}{*}{ G23 } & \multirow{2}{*}{6099} & KAUZ//ALTAR84/AOS/3/MILAN/KAUZ/4/SAUAL/5/SERI.1B//KAUZ \\
\hline & & /HEVO/3/AMAD*2/4/KIRITATI/6/KACHU/SAUAL \\
\hline \multirow{2}{*}{ G24 } & \multirow{2}{*}{6101} & KAUZ//ALTAR 84/AOS/3/MILAN/KAUZ/4/SAUAL/5/SERI.1B \\
\hline & & //KAUZ/ HEVO/3/AMAD*2/4/KIRITATI/6/KACHU/SAUAL \\
\hline \multirow{2}{*}{ G25 } & 6103 & KAUZ//ALTAR84/AOS/3/MILAN/KAUZ/4/SAUAL/5/PBW343*2/ \\
\hline & 0100 & KUKUNA//PARUS/3/PBW343*2/KUKUNA/6/KACHU/SAUAL \\
\hline G26 & 6104 & KAUZ//ALTAR84/AOS/3/MILAN/KAUZ/4/SAUAL/5/PBW343*2/ \\
\hline$G<0$ & 0104 & KUKUNA//PARUS/3/PBW343*2/KUKUNA/6/KACHU/SAUAL \\
\hline
\end{tabular}


Table 2. Contd.

\begin{tabular}{|c|c|c|}
\hline & & ATTILA/3*BCN//BAV92/3/TILHI/5/BAV92/3/PRL/SARA//TSI/VEE \\
\hline G27 & 6107 & $\begin{array}{l}\text { \#5/4/CROC_1/AE.SQUARROSA (224)//2*OPATA*2/6/ HUW234+ } \\
\text { LR34/PRINIA//UP2338*2/VIVITSI }\end{array}$ \\
\hline G28 & 139463 & unknown \\
\hline G29 & 6113 & $\begin{array}{l}\text { ROLF07*2/KIRITATI*2/10/PFAU/WEAVER }{ }^{*} 2 / / \text { BRAMBLING/9/RABE/6/WRM } \\
/ 4 / \text { FN/3*TH//K58/2*N/3/AUS-6869/5/ PELOTAS-ARTHUR/7/2* RABE/8/IRENA }\end{array}$ \\
\hline G30 & 6114 & $\begin{array}{l}\text { FRET2/KUKUNA//FRET2/3/YANAC/4/FRET2/KIRITATI²/5/WBLL1/ } \\
\text { KUKUNA//TACUPETO F2001/3/UP2338*2/VIVITSI }\end{array}$ \\
\hline G31 & 6115 & WBLL1*2/KUKUNA*2//WHEAR*2/3/ATTILA*2/PBW65*2//YANAC \\
\hline G32 & 6116 & $\begin{array}{l}\text { TRCH/SRTU//KACHU/3/WAXWING/PARUS//WAXWING/KIRITATI } \\
\text { /4/TRCH/SRTU//KACHU }\end{array}$ \\
\hline G33 & 6117 & $\begin{array}{l}\mathrm{PRL} / 2^{*} \mathrm{PASTOR} / / \mathrm{SRTU} / 3 / \mathrm{PRINIA} / \mathrm{PASTOR} / 5 / 2^{*} \mathrm{SERI} .1 \mathrm{~B} / / \mathrm{KAUZ} / \mathrm{HEVO} / 3 \\
\text { /AMAD*2/4/KIRITATI }\end{array}$ \\
\hline G34 & 6123 & $\begin{array}{l}\text { HW2045/3/WAXWING/SRTU//WAXWING/KIRITATI/4/KINGBIRD } \\
\text { \#1//INQALAB 91*2/TUKURU }\end{array}$ \\
\hline G35 & 6124 & $\begin{array}{l}\text { HW2045/3/WAXWING/SRTU//WAXWING/KIRITATI/4/KINGBIRD } \\
\text { \#1//INQALAB 91*2/TUKURU }\end{array}$ \\
\hline G36 & 41699 & unknown \\
\hline G37 & 6141 & $\begin{array}{l}\text { TC870344/GUI//TEMPORALERA M 87/AGR/3/2*WBLL1/5/ONIX } \\
\text { /4/MILAN/KAUZ//PRINIA/3/BAV92 }\end{array}$ \\
\hline G38 & 6145 & $\begin{array}{l}\text { W15.92/4/PASTOR//HXL7573/2*BAU/3/WBLL1/5/SOKOLL/3/PASTOR } \\
\text { //HXL7573/2*BAU }\end{array}$ \\
\hline G39 & 6148 & BECARD \#1/BAVIS \\
\hline G40 & 6149 & BECARD \#1/BAVIS \#1 \\
\hline G41 & 6155 & $\begin{array}{l}\text { VEE/MJI//2*TUI/3/PASTOR/4/BERKUT/6/2*OASIS/5*BORL95/5 } \\
\text { /CNDO/R143//ENTE/MEXI75/3/AE.SQ/4/2*OCl }\end{array}$ \\
\hline G42 & 6165 & BAVIS*2/4/PASTOR//HXL7573/2*BAU/3/SOKOLL/WBLL1 \\
\hline G43 & 80893 & unknown \\
\hline G44 & 6175 & ETBW115 (Digelu) \\
\hline G45 & 6180 & $\mathrm{KACHU} 2 / 3 / \mathrm{ND} 643 / / 2 * \mathrm{PRL} / 2^{*} \mathrm{PASTOR}$ \\
\hline G46 & 84772 & Unknown \\
\hline G47 & 6187 & $\begin{array}{l}\text { SHA7/VEE\#5//ARIV92/3/PBW343*2/KUKUNA/4/2*VARIS/MISR2, } \\
\text { EGY/3/FRET2/KUKUNA//FRET2 }\end{array}$ \\
\hline G48 & 6188 & $\begin{array}{l}\text { KACHU/SAUAL/4/VARIS/MISR2, EGY/3/FRET2/KUKUNA//FRET2 } \\
\text { /5/KACHU/SAUAL } \\
\text { VARIS/MISR2, EGY/3/FRET2/KUKUNA//FRET2*2/7/TUKURU// }\end{array}$ \\
\hline G49 & 6189 & $\begin{array}{l}\text { BAV92/RAYON/6/NG8201/KAUZ/4/SHA7//PRL/VEE\#6/3/FASAN/ } \\
\text { 5/MILAN/KAUZ }\end{array}$ \\
\hline G50 & 6192 & KRONSTAD F2004/3/TRCH/SRTU//KACHU/4/TRCH/SRTU//KACHU \\
\hline G51 & 6194 & $\begin{array}{l}\text { BABAX/LR42//BABAX/3/ER2000*2/4/SRN/AE.SQUARROSA (358)/ } \\
\text { / MILAN/SHA7 }\end{array}$ \\
\hline G52 & 6195 & KRONSTAD F2004/3/TRCH/SRTU//KACHU/4/TRCH/SRTU//KACHU \\
\hline G53 & 6199 & $\begin{array}{l}\text { KACHU/SAUAL/4/VARIS/MISR2, EGY/3/FRET2/KUKUNA//FRET2 } \\
\text { /5/ KACHU/SAUAL }\end{array}$ \\
\hline G54 & 6203 & $\begin{array}{l}\text { KACHU/SAUAL/4/VARIS/MISR2, EGY/3/FRET2/KUKUNA//FRET2 } \\
\text { /5/KACHU/SAUAL }\end{array}$ \\
\hline G55 & 120699 & Unknown \\
\hline G56 & 6207 & $\begin{array}{l}\text { FRET2/KUKUNA//FRET2/3/WHEAR/4/FRET2*2/KUKUNA/5/2* } \\
\text { WBLL1/KUKUNA//TACUPETO F2001/3/UP2338*2/VIVITSI }\end{array}$ \\
\hline G57 & 6208 & $\begin{array}{l}\text { FRET2/KUKUNA//FRET2/3/WHEAR/4/FRET2*2/KUKUNA/5/2* } \\
\text { WBLL1/KUKUNA//TACUPETO F2001/3/UP2338*2/VIVITSI }\end{array}$ \\
\hline G58 & DANDAA & KIRITATI//2*PBW65/2*SERI.IB \\
\hline G59 & PBW343 & Unknown \\
\hline G60 & WANE & SOKOLL/EXCALIBUR \\
\hline
\end{tabular}


was applied at planting while urea was split into half at planting and the remaining half at tillering stage. The plants were repeatedly irrigated to ensure optimum plant growth and development, create favourable environment for disease development and enable the plants to express their genetic resistance to stem rust (Kosgey et al., 2015; Nzuve et al., 2013).

\section{Data collection}

\section{Seedling resistance test}

Disease data was collected 14 days after inoculation using Stakman et al. (1962) scoring system ( IT = $0-4$ ) with McIntosh et al. (1995) modifications: where ITs "0" represented no visible uredinia, ";" (hypersensitive flecks without uredinia), "1" (small uredinia surrounded by necrosis), "2" (small to medium sized uredinia usually with green islands and surrounded by chlorosis or necrosis), " 3 " (moderate sized uredinia with or without chlorosis) and " 4 " (large uredinia without chlorosis). The plus and minus signs were used to indicate the occurrence of larger or smaller uredinia than the normal uredinia, respectively within a given Infection type. Genotypes with low infection types (ITs=0-2) were considered as resistant, and genotypes with infection type $=2+$ and 3 - showed mixed reaction (resistance and susceptible) while genotypes with high infection types $(\mathrm{ITs}=3-4)$ were considered as susceptible for stem rust disease.

\section{Adult plant resistance test}

Rust severity data collection was started when the spreader row plants showed maximum rust infection and continued at a weekly interval up to the plants attaining physiological maturity. Rust severity was determined as percentage using Peterson et al. (1948) modified Cobbs' scale method. The response of plants to stem rust infection was measured based on the pustule size and any associated necrotic and/or chlorotic lesions that occurred on plants at field conditions. According to Roelfs et al. (1992) scaling method, the response of plants to stem rust infection were classified as follows: $R=$ resistant, $R M R=$ resistant to moderately resistant, $\mathrm{MR}=$ moderately resistant, MRMS = moderately resistant to moderately susceptible, MSS= moderately susceptible to susceptible, MS = moderately susceptible, and $S=$ susceptible. Selected quantitative traits such as Days to heading, Days to maturity, Thousands kernels weight $(\mathrm{g})$, Hectoliter weight $(\mathrm{g} / \mathrm{hL}$ ) and grain yield (adjusted at moisture content $12.5 \%$ ) were also collected by considering all plants of each plot as sample while data of Plant height $(\mathrm{cm})$, Spike length $(\mathrm{cm})$, Number of spikelets per spike and Number of kernels per spike were collected from five randomly selected plants from each plot.

\section{Data analysis}

The generated data were subjected to separate analysis of variance for each location using Genstat software 13th edition (Payne et al., 2011) to observe differences among genotypes on their resistance to stem rust. Bartlett's chisquare test and normality test were also used to check the homogeneity and the normal distribution of error variance between environments, respectively. Combined analysis of variance over locations was conducted using linear mixed model. The genotypes were considered as a fixed effect while blocks, replications and environments were random effects. The least significant difference (LSD) value was used to compare genotypic means at $p<0.05$ probability level. The ANOVA was fitted as the following linear Mathematical model:

$\boldsymbol{y}_{i j k l m}=\mu+\boldsymbol{\rho}_{i}+\boldsymbol{\iota}_{j}+\boldsymbol{b}_{m(\iota)}+\boldsymbol{\rho} \boldsymbol{\iota}_{j i}+\boldsymbol{\varepsilon}_{i j k l m}$

Where, $\boldsymbol{y}_{i k k m}=$ the observed value for the $i^{\text {th }}$ genotype from $j^{\text {th }}$ location, $m^{\text {th }}$ block nested within $f^{\text {th }}$ replication; $\boldsymbol{\mu}=$ the general mean effect, $\boldsymbol{\rho}_{i}=$ the $i^{\text {th }}$ genotype effect (considered as fixed effect), $\boldsymbol{\iota}_{j}=$ the $j^{\text {th }}$ environment effect (considered as random effect), $\boldsymbol{b}_{m(1)}=$ the effect of $m^{\text {th }}$ replication nested within the $i^{\text {th }}$ environment, $\quad \boldsymbol{\rho}_{j i}=$ interaction effect of $f^{\text {th }}$ environment and $i^{\text {th }}$ genotype (considered as random effect) and $\boldsymbol{\varepsilon}_{i j k l m}=$ the experimental error(considered as random). Besides, area under disease progress curve (AUPDC) of each genotype was also calculated by using Wilcox son et al. (1975) method.

AUDPC $=\sum_{i=1}^{n}\left[\left\{\frac{\left(X_{i}+X_{i+1}\right)}{2}\right\}\left(t_{i+1}-t_{i}\right)\right]$

Where $x_{i}=$ stem rust severity on the $i^{\text {th }}$ date, $t_{i}=$ the time in days after appearance of the disease, and $n=$ number of date on which stem rust will be recorded. Spearman rank correlation coefficient was also carried out to know the relationship between disease measuring parameters. Coefficient of infection $(\mathrm{Cl})$ was also calculated by multiplying the rust severity with constant value for field response where $R=0, R M R=0.1, M R=0.2, M R M S=0.4$, $\mathrm{MS}=0.6, \mathrm{MSS}=0.8$ and $S=1$ (Stubbs et al., 1986). Apparent infection rate $(r)$ was calculated by using vander Plank's equation (vander Plank, 1963) as follows:

$r=\frac{1}{\left(t_{2}-t_{1}\right)}\left[\log _{e}^{\frac{x_{2}}{1-x_{1}}}-\log _{e}^{\frac{x_{1}}{1-x_{1}}}\right]$

Where: "r" = Apparent infection rate; " $t$ " = initial time of disease assessment; " $\mathrm{t}_{2}$ " = final time of disease assessment; " $\mathrm{x}_{1}$ " and " $\mathrm{x}_{2}$ " represent amounts of disease present at " $\mathrm{t}_{1}$ " and " $\mathrm{t}_{2}$ " respectively. The principal component analysis was calculated using disease measuring parameters and other agronomic traits through varimax rotation method that is generally considered 
Table 3. Seedling infection type (IT) for seedling test against race TTKSK.

\begin{tabular}{lcl}
\hline Infection type & Number of genotypes & Genotypes reaction to race TTKSK \\
\hline 2 & 32 & Resistance reaction \\
$2+3-$ & 41 & Mixed reaction \\
$3-$ & 17 & Moderate Susceptible reaction \\
3 & 25 & Susceptible reaction \\
$3+$ & 5 & Very Susceptible reaction \\
\hline
\end{tabular}

Table 4. Analysis of variance for FRS, AUDPC and Cl of genotypes at individual locations.

\begin{tabular}{|c|c|c|c|c|c|c|c|c|c|c|}
\hline \multirow[b]{2}{*}{ sov } & \multirow{2}{*}{ df } & \multicolumn{3}{|c|}{ Debrezeit } & \multicolumn{3}{|c|}{ Adet } & \multicolumn{3}{|c|}{ Kulumsa } \\
\hline & & FRS & $\mathrm{Cl}$ & AUDPC & FRS & $\mathrm{Cl}$ & AUDPC & FRS & $\mathrm{Cl}$ & AUDPC \\
\hline Rep & 2 & $342^{\star \star \star}$ & $121.8^{* * t}$ & $18418^{\star \star \star}$ & $1799.7^{\star \star \star}$ & $275.47^{\star \star \star}$ & $40981.3^{* \star \star}$ & $1830.1^{* \star \star}$ & $533.1^{* t * t}$ & $89515^{\star \star \star}$ \\
\hline Rep/Block & 8 & 14.38 ns & $8.273^{\mathrm{ns}}$ & $1390 \mathrm{~ns}$ & $9.62 \mathrm{~ns}$ & $1.56^{\mathrm{ns}}$ & $185.2^{\mathrm{ns}}$ & $67.5^{\mathrm{ns}}$ & $12.47^{\mathrm{ns}}$ & 2359 ns \\
\hline Genotypes & 59 & $665.8^{* * *}$ & $186.6^{* * *}$ & $34827.3^{\star * *}$ & $287.96^{* \star *}$ & $173.92^{\star \star \star}$ & $40028.2^{* * *}$ & $446.7^{\star \star \star}$ & $169.8^{* * t}$ & $33933.3^{* * *}$ \\
\hline Residual & 110 & 23.78 & 8.64 & 1288 & 16.97 & 4.20 & 578.8 & 84.5 & 14.55 & 2285 \\
\hline LEE & 84 & 23.14 & 8.62 & 1295.7 & 16.46 & 4.02 & 551.81 & 83.34 & 14.41 & 2289.7 \\
\hline
\end{tabular}

${ }^{*},{ }^{* *},{ }^{* * *}$ Significant at $0.05,0.01$ and 0.001 probability levels, respectively; $\mathrm{ns}=$ non-significant, SOV $=$ sources of variation, $\mathrm{df}=\mathrm{Degree}$ of freedom, $\mathrm{E}=$ Environment, $\mathrm{G}=$ Genotype, LEE=Lattice effective error, FRS= Final rust severity, $\mathrm{Cl}=$ coefficient infection, AUDPC= Area under Disease Prog ress Curve, $r=$ Apparent infection rate.

superior to other orthogonal factor rotation methods in achieving a simplified factor structure (Hair et al., 2010). Hierarchical cluster analysis was carried out by using SAS software for windows 9.

\section{RESULTS AND DISCUSION}

\section{Seedling reaction}

Among 120 tested genotypes evaluated in greenhouse, 32 (26.67\%) of the genotypes showed seedling infection type $(\mathrm{IT}=2)$ with small to medium sized uredinia usually with green islands and surrounded by chlorosis or necrosis as shown Table 3. This indicated that those genotypes were resistant to race TTKSK due to the presence of race specific major resistant gene which was expressed at seedling stage of genotypes. While $41(34.17 \%)$ of the tested genotypes exhibited mixed reaction (2+3-) which suggesting that these genotypes would have minor non race specific resistance genes that able to stay for a longer period of time without losing their resistance. Majority 47 $(39.16 \%)$ of the tested genotypes showed susceptible seedling reaction (IT=3- to $3+$ ) with moderate sized uredinia and with or without chlorosis. Similarly, different authors (Hundie et al., 2018; Olivera, et al., 2018) reported the existence of variability among bread wheat genotypes on their response to different stem rust races at their seedling stage.

\section{Adult plant resistance performance of genotypes at individual locations}

Analysis of variance for FRS, AUDPC and $\mathrm{Cl}$ at individual location are presented in Table 4. The result of ANOVA revealed the presence of highly significant $(p<0.001)$ variation among genotypes on the traits of FRS, AUDPC and $\mathrm{Cl}$ at each of the three individual testing locations. This indicated that the presence of a considerable amount of genetic variation among the tested genotypes. The mean values of final stem rust severity (FS), coefficient of infection $(\mathrm{Cl})$, area under disease progress curve (AUDPC), apparent infection rate ( $r$ ) and host response (HR) of 60 bread wheat genotypes at Debrezeit, Adet and Kulmsa locations are presented in Table 5. There was variation in plants response to stem rust disease at field conditions. These responses were ranging from MRMS that exhibited as small/medium uredia coupled with either chlorotic or necrotic areas to susceptible reaction which display as large uredia present, generally with little or no chlorosis and necrosis. Significant differences were also observed among the bread wheat genotypes for $\mathrm{FS}, \mathrm{Cl}$, AUDPC and $r$ at individual location. This showed the presence of great variation among the tested bread wheat genotypes on their resistance potential against the race Ug99. The highest overall mean values of genotypes in FRS (29.61), Cl (11.26) and AUDPC (149.4) were recorded at Debrezeit testing location. This is due to the combination of moderate temperature and wet environmental conditions at Deberezit $\left(28.3^{\circ} \mathrm{C}, 851 \mathrm{~mm}\right)$ was more favourable to rust development than the other two testing locations; thus, high rust disease pressure overcame the defense system of the plants in Debrezeit conditions as previously reported by Agrios (2005). G12 and G59 also scored the highest (65) and lowest (5) FRS values at both Adet $(50,5)$ and Kulmsa $(65,5)$ locations, respectively. 
Table 5. Mean values of FRS, CI, AUDPC, r, HR and IT for the 60 Bread genotypes grown at individual locations.

\begin{tabular}{|c|c|c|c|c|c|c|c|c|c|c|c|c|c|c|c|c|}
\hline \multirow{2}{*}{ Geno } & \multicolumn{5}{|c|}{ Debrezeit } & \multicolumn{5}{|c|}{ Adet } & \multicolumn{5}{|c|}{ Kulmsa } & \multirow{2}{*}{$\begin{array}{l}\text { Infection } \\
\text { Type (IT) }\end{array}$} \\
\hline & FRS & $\mathrm{Cl}$ & AUDPC & $r$ & HR & FRS & $\mathrm{Cl}$ & AUDPC & $r$ & HR & FRS & $\mathrm{Cl}$ & AUDPC & $r$ & HR & \\
\hline G1 & 23.3 & 7.22 & 94.5 & 0.054 & MR-MS & 21.7 & 3.22 & 35.8 & 0.094 & MRMS & 18.3 & 2.78 & 31.8 & 0.072 & MR-MS & $2+3-$ \\
\hline $\mathrm{G} 2$ & 69.3 & 31.00 & 408.0 & 0.165 & $\mathrm{~S}$ & 47.7 & 27.00 & 405.0 & 0.155 & $\mathrm{~S}$ & 61.0 & 28.00 & 409.0 & 0.153 & $\mathrm{~S}$ & 3 \\
\hline G3 & 30.0 & 20.67 & 303.3 & 0.105 & $S$ & 20 & 5.11 & 60.7 & 0.202 & MS & 36.7 & 23.89 & 327.8 & 0.046 & $S$ & 3 \\
\hline G4 & 21.7 & 6.22 & 86.3 & 0.086 & MR-MS & 20 & 10.29 & 134.9 & 0.091 & $S$ & 18.3 & 10.45 & 144.7 & 0.070 & MS-S & $2+3-$ \\
\hline G5 & 20.0 & 6.33 & 79.3 & 0.187 & MS-S & 21.7 & 6.11 & 74.7 & 0.219 & MS-S & 23.3 & 9.67 & 131.8 & 0.251 & MS-S & $2+3-$ \\
\hline G6 & 18.3 & 5.89 & 79.3 & 0.081 & MS & 15 & 5.56 & 78.2 & 0.110 & MS & 25.0 & 7.33 & 94.5 & 0.167 & MS & 3- \\
\hline G7 & 35.0 & 10.75 & 133.9 & 0.152 & MS & 20 & 5.91 & 66.8 & 0.127 & MS-S & 28.3 & 9.11 & 122.5 & 0.233 & MS & 3- \\
\hline G8 & 18.3 & 7.00 & 91.0 & 0.047 & MS-S & 21.7 & 7.02 & 87.7 & 0.116 & MS & 20.0 & 8.78 & 117.8 & 0.106 & MS-S & $2+3-$ \\
\hline G9 & 15.0 & 5.56 & 75.8 & 0.058 & MS & 31.7 & 21.11 & 309.2 & 0.111 & $S$ & 28.3 & 13.34 & 178.5 & 0.253 & $S$ & $3-$ \\
\hline G10 & 18.3 & 6.27 & 83.3 & 0.103 & MS & 16.7 & 2.78 & 35.0 & 0.033 & MR-MS & 13.3 & 4.11 & 56.0 & 0.027 & MR-MS & 3- \\
\hline G11 & 53.0 & 27.00 & 375.0 & 0.105 & MS-S & 43.7 & 23.0 & 364.0 & 0.090 & MS & 48.3 & 25.00 & 367.2 & 0.101 & MS-S & $3+$ \\
\hline G12 & 5.0 & 0.31 & 3.6 & 0.000 & MR-MS & 5.0 & 0.29 & 4.3 & 0.060 & MR-MS & 5.0 & 0.51 & 7.3 & 0.093 & MR-MS & $2+3-$ \\
\hline G13 & 20.0 & 7.33 & 98.0 & 0.064 & MS-S & 18.3 & 5.69 & 77.2 & 0.136 & MS & 21.7 & 5.45 & 70.0 & 0.054 & MS & $2+3-$ \\
\hline G14 & 20.0 & 6.00 & 79.3 & 0.085 & MR-MS & 16.7 & 3.41 & 46.3 & 0.123 & MR-MS & 21.7 & 4.58 & 65.6 & 0.134 & MR-MS & $2+3-$ \\
\hline G15 & 71.7 & 30.00 & 432.0 & 0.172 & $S$ & 48.3 & 27.0 & 408.0 & 0.164 & $\mathrm{~S}$ & 63.0 & 30.00 & 412.0 & 0.168 & $\mathrm{~S}$ & 3 \\
\hline G16 & 50.0 & 20.00 & 261.3 & 0.083 & MS-S & 26.7 & 9.22 & 121.3 & 0.226 & MS-S & 33.3 & 10.33 & 138.8 & 0.251 & MS & 3 \\
\hline G17 & 36.7 & 14.22 & 180.8 & 0.056 & MS-S & 20 & 7.15 & 89.1 & 0.086 & MS-S & 23.3 & 8.45 & 119.0 & 0.234 & MS & $2+3-$ \\
\hline G18 & 30.0 & 8.67 & 107.3 & 0.123 & MS & 15 & 3.33 & 46.7 & 0.177 & MR-MS & 23.3 & 5.61 & 82.3 & 0.184 & MR-MS & $3-$ \\
\hline G19 & 33.3 & 10.78 & 134.2 & 0.119 & MS & 6.7 & 1.32 & 15.4 & 0.017 & MS & 20.0 & 7.22 & 102.7 & 0.145 & MS & 3- \\
\hline G20 & 20.0 & 7.64 & 101.3 & 0.152 & MS-S & 21.7 & 7.35 & 91.2 & 0.133 & MS-S & 30.0 & 8.67 & 102.7 & 0.177 & MS-S & $2+3-$ \\
\hline G21 & 38.3 & 9.86 & 124.6 & 0.169 & MS & 30 & 15.00 & 213.5 & 0.094 & MS-S & 26.7 & 10.89 & 151.7 & 0.235 & MS-S & 3- \\
\hline G22 & 43.3 & 14.07 & 172.2 & 0.077 & MS-S & 30 & 13.29 & 162.9 & 0.078 & $S$ & 38.3 & 18.78 & 252.0 & 0.167 & $S$ & 3 \\
\hline G23 & 40.0 & 22.22 & 326.7 & 0.106 & MS-S & 26.7 & 10.00 & 133.0 & 0.240 & MS-S & 41.7 & 16.67 & 221.7 & 0.174 & MS-S & $2+3-$ \\
\hline G24 & 30.0 & 13.07 & 179.2 & 0.126 & MS-S & 30 & 7.81 & 99.5 & 0.208 & MS & 28.3 & 8.45 & 119.0 & 0.251 & MS & $2+3-$ \\
\hline G25 & 20.0 & 10.11 & 141.2 & 0.066 & MS-S & 16.7 & 6.00 & 84.0 & 0.075 & MS & 23.3 & 9.78 & 136.5 & 0.073 & MS & $2+3-$ \\
\hline G26 & 38.3 & 18.67 & 270.7 & 0.137 & MS-S & 26.7 & 7.44 & 92.2 & 0.207 & MS & 33.3 & 11.89 & 150.5 & 0.161 & MS-S & 3 \\
\hline G27 & 23.3 & 7.67 & 99.2 & 0.088 & MS-S & 16.7 & 3.70 & 52.9 & 0.188 & MRMS & 16.7 & 4.39 & 60.1 & 0.094 & MR-MS & $2+3-$ \\
\hline G28 & 26.7 & 8.11 & 110.8 & 0.094 & MS & 18.3 & 4.15 & 52.9 & 0.161 & MR-MS & 11.7 & 2.28 & 33.3 & 0.122 & MR-MS & 3- \\
\hline G29 & 28.3 & 9.02 & 108.7 & 0.163 & MS-S & 20 & 6.10 & 75.3 & 0.152 & MS-S & 30.0 & 11.44 & 157.5 & 0.207 & MS-S & $2+3-$ \\
\hline G30 & 23.3 & 6.34 & 87.5 & 0.132 & MR-MS & 15 & 3.69 & 48.1 & 0.133 & MR-MS & 15.0 & 1.47 & 19.20 & 0.027 & MR-MS & $2+3-$ \\
\hline G31 & 6.7 & 0.87 & 11.6 & 0.055 & MR-MS & 6.7 & 0.50 & 7.3 & 0.110 & MR-MS & 10.0 & 1.47 & 19.2 & 0.027 & MR-MS & $2+3-$ \\
\hline G32 & 23.3 & 7.78 & 105.0 & 0.067 & MS-S & 21.7 & 5.72 & 74.1 & 0.226 & MS & 25.0 & 8.44 & 107.3 & 0.287 & MS-S & $2+3-$ \\
\hline G33 & 20.0 & 5.34 & 70.1 & 0.106 & MS & 21.7 & 5.00 & 68.8 & 0.078 & MRMS & 21.7 & 4.67 & 60.7 & 0.084 & MS & 3- \\
\hline G34 & 38.3 & 13.49 & 166.1 & 0.103 & MS-S & 30 & 10.73 & 133.7 & 0.084 & MS-S & 35.0 & 24.33 & 337.2 & 0.046 & MS-S & 3 \\
\hline G35 & 43.3 & 14.23 & 174.0 & 0.171 & MS & 30 & 9.41 & 112.9 & 0.224 & MS-S & 33.3 & 11.17 & 142.9 & 0.195 & MS-S & $2+3-$ \\
\hline G36 & 26.7 & 9.31 & 116.4 & 0.139 & MS-S & 23.3 & 8.20 & 100.8 & 0.146 & MS-S & 33.3 & 10.73 & 127.9 & 0.056 & MS-S & $2+3-$ \\
\hline G37 & 36.7 & 13.98 & 178.3 & 0.140 & MS-S & 31.7 & 20.56 & 291.7 & 0.082 & $\mathrm{~s}$ & 23.3 & 12.00 & 166.8 & 0.037 & MS-S & 3 \\
\hline G38 & 26.7 & 9.44 & 130.7 & 0.325 & MS & 21.7 & 6.86 & 93.1 & 0.135 & MS & 28.3 & 19.22 & 273.0 & 0.086 & $S$ & 3- \\
\hline G39 & 20.0 & 8.22 & 107.3 & 0.034 & MS-S & 21.7 & 7.02 & 95.9 & 0.168 & MS & 23.3 & 6.33 & 87.5 & 0.095 & MR-MS & $2+3-$ \\
\hline
\end{tabular}


Table 5. Contd.

\begin{tabular}{|c|c|c|c|c|c|c|c|c|c|c|c|c|c|c|c|c|}
\hline G40 & 30.0 & 10.76 & 137.4 & 0.160 & MS-S & 41.7 & 26.67 & 373.3 & 0.072 & $S$ & 30.0 & 13.45 & 178.5 & 0.214 & $S$ & $2+3-$ \\
\hline G41 & 23.3 & 4.57 & 60.8 & 0.140 & MR-MS & 20 & 3.63 & 47.5 & 0.194 & MR-MS & 28.3 & 13.33 & 164.5 & 0.034 & $S$ & $2+3-$ \\
\hline G42 & 20.0 & 7.98 & 108.3 & 0.170 & MS-S & 21.7 & 5.74 & 74.4 & 0.224 & MS & 23.3 & 8.82 & 123.0 & 0.136 & MS & $2+3-$ \\
\hline G43 & 30.0 & 7.78 & 100.3 & 0.071 & MR-MS & 20 & 4.22 & 56.0 & 0.067 & MR-MS & 26.7 & 14.11 & 186.7 & 0.072 & $S$ & $2+3-$ \\
\hline G44 & 65.0 & 30.00 & 403.0 & 0.135 & MS-S & 40 & 25 & 396.0 & 0.125 & MS-S & 51.7 & 26.00 & 399.0 & 0.128 & $S$ & 3- \\
\hline G45 & 38.3 & 14.89 & 191.3 & 0.071 & MS-S & 21.7 & 9.56 & 131.8 & 0.014 & MS & 30.0 & 19.56 & 277.7 & 0.070 & $S$ & $3+$ \\
\hline G46 & 15.0 & 6.44 & 85.2 & 0.048 & MS-S & 18.3 & 5.93 & 79.8 & 0.191 & MSS & 26.7 & 10.11 & 129.5 & 0.123 & MS & $2+3-$ \\
\hline G47 & 20.0 & 6.33 & 89.8 & 0.230 & MS-S & 18.3 & 3.89 & 54.8 & 0.204 & MR-MS & 26.7 & 12.89 & 177.3 & 0.085 & MS-S & $2+3-$ \\
\hline G48 & 30.0 & 10.22 & 130.7 & 0.067 & MS-S & 15 & 3.36 & 44.6 & 0.216 & MR-MS & 16.7 & 3.44 & 47.8 & 0.194 & MR-MS & $2+3-$ \\
\hline G49 & 36.7 & 17.75 & 241.3 & 0.164 & MS-S & 30 & 9.44 & 116.7 & 0.208 & MS-S & 40.0 & 17.85 & 228.2 & 0.102 & $S$ & $2+3-$ \\
\hline G50 & 30.0 & 8.57 & 115.6 & 0.166 & MS & 30 & 5.27 & 67.4 & 0.166 & MR-MS & 28.3 & 5.33 & 72.3 & 0.276 & MR-MS & $3-$ \\
\hline G51 & 26.7 & 8.11 & 101.5 & 0.116 & MS & 30 & 8.44 & 102.7 & 0.187 & MS & 26.7 & 5.60 & 66.3 & 0.193 & MS & 3- \\
\hline G52 & 8.3 & 1.59 & 20.8 & 0.110 & MR-MS & 8.3 & 0.69 & 10.2 & 0.110 & MR-MS & 10.0 & 2.17 & 28.6 & 0.050 & MR-MS & $2+3-$ \\
\hline G53 & 25.0 & 9.09 & 116.4 & 0.142 & MS-S & 26.7 & 7.56 & 93.3 & 0.190 & MS-S & 33.3 & 12.00 & 151.7 & 0.144 & MS-S & $2+3-$ \\
\hline G54 & 26.7 & 8.68 & 105.1 & 0.149 & MS-S & 16.7 & 5.00 & 60.0 & 0.144 & MS-S & 26.7 & 7.93 & 90.8 & 0.138 & MS-S & $2+3-$ \\
\hline G55 & 20.0 & 7.27 & 90.3 & 0.076 & MS-S & 18.3 & 6.15 & 85.6 & 0.257 & MS-S & 23.3 & 8.17 & 109.1 & 0.211 & MS-S & $2+3-$ \\
\hline G56 & 21.7 & 4.28 & 54.3 & 0.044 & MR-MS & 18.3 & 4.05 & 49.6 & 0.067 & MR-MS & 28.3 & 4.67 & 61.8 & 0.094 & MR-MS & $2+3-$ \\
\hline G57 & 21.7 & 5.89 & 82.8 & 0.095 & MR-MS & 18.3 & 4.67 & 65.3 & 0.068 & MR-MS & 20.0 & 5.33 & 74.7 & 0.088 & MR-MS & $2+3-$ \\
\hline G58 & 48.0 & 28.00 & 380.0 & 0.128 & MS & 35.0 & 24.00 & 372.0 & 0.116 & MS & 41.7 & 8.71 & 376.0 & 0.120 & MS & 3 \\
\hline G59 & 73.0 & 36.33 & 456.0 & 0.173 & $S$ & 50.0 & 31.33 & 416.0 & 0.168 & $S$ & 65.0 & 33.0 & 420.0 & 0.175 & MR-MS & $3+$ \\
\hline G60 & 5.0 & 0.51 & 7.4 & 0.055 & MR-MS & 6.7 & 0.38 & 4.9 & 0.055 & MR-MS & 8.3 & 0.58 & 8.1 & 0.072 & MR-MS & $2+3-$ \\
\hline Mean & 29.61 & 11.26 & 149.4 & 0.114 & & 23.36 & 8.80 & 120.8 & 0.139 & & 28.01 & 10.68 & 148.68 & 0.142 & & \\
\hline LSD & 7.77 & 4.75 & 58.2 & 0.096 & & 6.56 & 3.25 & 37.9 & 0.095 & & 14.92 & 6.14 & 77.37 & 0.111 & & \\
\hline $\mathrm{CV}(\%)$ & 16.2 & 26.0 & 24.1 & 52.3 & & 17.38 & 22.82 & 19.45 & 42.16 & & 33.0 & 34.6 & 32.19 & 48.3 & & \\
\hline
\end{tabular}

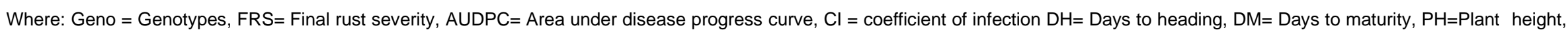

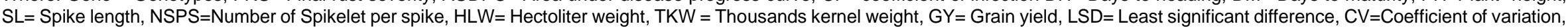
MR-MS = moderately resistant to moderately susceptible, $M S=$ moderately susceptible, MS-S = moderately susceptible to susceptible and $\mathrm{S}=$ susceptible .

In the case of AUDPC values, the maximum (456) and minimum (3.6) values across the three locations were scored by G59 and G12, respectively. Moreover, the magnitude apparent infection rate of the genotypes ranged between 0.000 to 0.325 at Debrezeit; and also ranged from 0.017 to 0.257 and 0.027 to 0.287 at Adet and Kulmsa locations, respectively. The smaller range value of coefficient of infection ( $r$ ) recorded at Adet indicated the occurrence of a more steady lower disease infection progression than the other two locations. Generally, identification of susceptible or resistant genotypes based on " $r$ " values alone may not lead to useful results. Hence, it is paramount to use two or more disease index parameters to delineate the genotypes whether they are susceptible or resistance to stem rust disease. Genotypes such as G59, G15, G2, G44, G11 and G58 had scores (FRS $\geq 35$, AUDPC $>360, \mathrm{Cl}>20$ and $r>0.10$ ) with susceptible plant response across the three locations and considered as very susceptible genotypes; whereas G12, G60, G31 and G52 were found with (FRS $\leq 10, A U D P C<30$, $\mathrm{Cl}<3$ and $\mathrm{r}<0.2$ ) values and considered as resistant genotypes. This indicated that these selected genotypes would have gene Sr13 which is effective against pathotype TTKSK and its derivatives; and could be used as source of slow rusting resistance gene in wheat breeding research program. In their previous studies, different authors (Safavi et al., 2013; Draz et al., 2015) used FRS, Cl and AUDPC for measuring the slow rusting resistance potential of wheat genotypes under field conditions. 
Table 6. Mean sum of square for ACI, AUDPC and Agronomic traits of 60 wheat genotypes across locations.

\begin{tabular}{|c|c|c|c|c|c|c|c|c|c|c|c|c|c|}
\hline sov & df & FRS & $\mathrm{Cl}$ & AUDPC & $r$ & DH & DM & PH & SL & NSPS & HLW & TKW & GY \\
\hline Genotypes & 59 & $1252.85^{* * *}$ & $468.48^{* * *}$ & $94816.30^{* * *}$ & $0.0179^{* * *}$ & $90.70^{* * *}$ & $368.96^{* * *}$ & $320.16^{* * *}$ & $4.12^{* * *}$ & $152.07^{* * *}$ & $94.94^{* * *}$ & $176.10^{* * *}$ & $1.90^{* *}$ \\
\hline Environment & 2 & $1886.12^{\mathrm{ns}}$ & $930.66^{*}$ & $148914.3^{*}$ & $0.0431^{\mathrm{ns}}$ & $488.41^{\mathrm{ns}}$ & $6621.80^{*}$ & $212.33^{\text {ns }}$ & 0.459 ns & $5461.78^{* *}$ & $1106.49^{* * *}$ & $529.14^{\mathrm{ns}}$ & $51.38^{* * *}$ \\
\hline Env.Rep & 6 & $1323.93^{* * *}$ & $108.29^{*}$ & $16014.45^{\mathrm{NS}}$ & $0.0326^{* *}$ & $221.89^{* * *}$ & $892.87^{* * *}$ & $334.98^{* * *}$ & $3.72^{*}$ & $19.03^{* * *}$ & $14.48^{\text {ns }}$ & $328.28^{* * *}$ & $3.05^{* * *}$ \\
\hline Env.Rep.Block & 36 & $109.52^{* * *}$ & $43.56^{* * *}$ & $9835.87^{* * *}$ & $0.0094^{\star \star \star}$ & $11.344^{* * *}$ & $44.72^{* * *}$ & $67.69^{* * *}$ & $0.95^{* \star *}$ & $152.06^{* * *}$ & $12.27^{* * *}$ & $42.36^{* * *}$ & $0.30^{* *}$ \\
\hline GXE & 118 & $82.66^{* * *}$ & $31.84^{* * *}$ & $6567.17^{* * *}$ & $0.0084^{\star \star *}$ & $7.00^{* * *}$ & $29.89^{* * *}$ & $81.52^{* * *}$ & $0.24^{\mathrm{ns}}$ & $9.00^{* * *}$ & $8.13^{* * *}$ & $18.99^{* * *}$ & $0.26^{* * *}$ \\
\hline Error & 318 & 33.23 & 5.12 & 421.62 & 0.0033 & 0.0154 & 0.287 & 13.28 & 0.194 & 3.098 & 3.171 & 11.59 & 0.159 \\
\hline
\end{tabular}

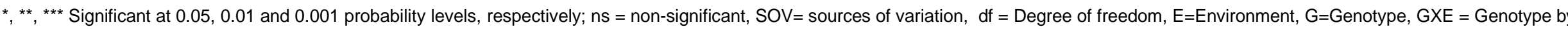

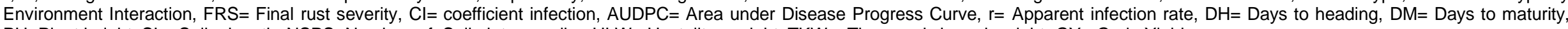
$\mathrm{PH}=$ Plant height, $\mathrm{SL}=$ Spike length, NSPS=Number of Spikelet per spike, HLW= Hectoliter weight, TKW = Thousands kernel weight, GY= Grain Yield.

\section{Adult plant resistance performance of bread genotypes over the three locations}

The combined analysis of variance for FRS, $\mathrm{Cl}$, AUDPC, $r$ and other agronomic traits across the three locations are presented in Table 6 . The results showed highly significant differences $(\mathrm{p}<$ 0.001 ) among genotypes in FRS, Cl, AUDPC, $r$ and in all other agronomic traits. This indicated the existence of sufficient variability among genotypes on their response to stem rust disease which caused by race Ug99. Moreover, highly significant differences ( $p<0.001)$ on GY and HLW; and significant differences on $\mathrm{Cl}$, AUDPC, DM and NSPS were also observed among locations. This suggested that testing locations had considerable effects on rust disease resistance and agronomic performance of genotypes. It also indicated the existence of different stem rust disease pressures across the testing locations. The existence of highly significant $(p<0.001)$ genotype by location $(G \times E)$ interaction effects on FRS, Cl, AUDPC, r, $\mathrm{DH}, \mathrm{DM}, \mathrm{PH}, \mathrm{HLW}, \mathrm{TKW}$ and GY performances of genotypes indicated that inconsistent performance of genotypes across the three locations. This explained the extent of challenges that farmers would face when they try to manage stem rust disease by using improved variety that has race specific resistance gene since this gene might not work in areas where a virulent race does not existed. Furthermore, this result also implying the importance of location specific breeding program; different varieties have to be developed for different environments (Acquaah, 2007).

The combined mean performances of both disease measuring and agronomic traits across locations indicated that the highest (62.5) and lowest (5.0) scores of FRS were recorded by G59 and $\mathrm{G} 12$ respectively as shown in Table 7 . Besides, G59 and G12 had also scored the maximum (430.7) and minimum (5.1) AUDPC values of genotypes across locations, respectively. This implied that the two genotypes have consistent stem rust resistance performance across the testing locations, therefore, farmers can use G12 for commercial production of wheat across the three locations. In the case of yield and yield related traits, G39 showed the highest scores of NSPS (41.4 spikelet/spike), TKW (47.3g) and GY (4.7t/ha) across the three locations. Therefore, G39 can be used as a parent material for future breeding program to improve NSPS, TKW and GY of bread wheat genotypes.

\section{Interrelationships among stem rust disease traits}

The results of correlation analysis among the stem rust disease measuring parameters are presented in Table 8. A positive and highly significant correlation of FRS with $\mathrm{Cl}\left(\mathrm{r}^{2}=0.894\right)$ and AUDPC $\left(r^{2}=0.877\right)$ implied that severity of stem rust disease increased with increasing of $\mathrm{Cl}$ and AUDPC. This significant positive correlation could have resulted from the effect of strong coupling linkage between genes or due to pleiotropic genes that control these traits in the same direction. The results also indicated that the FRS values of genotypes at adult plant test could, therefore, be used to extrapolate information about the $\mathrm{Cl}$ and AUDPC values of the tested genotypes with a better accuracy. Numerous authors (Safavi et al., 2010; Duncan et al., 2015) used FRS, Cl and AUDPC to identify bread wheat genotypes that have partial resistance to stem rust disease. Weak and non-significant positive correlations were observed between apparent infection rate $(r)$ and 
Table 7. Mean performances of genotypes on FRS, $\mathrm{Cl}$, AUDPC and selected agronomic traits across locations.

\begin{tabular}{|c|c|c|c|c|c|c|}
\hline Geno & FRS & $\mathrm{Cl}$ & AUDPC & NSPS & TKW & GY \\
\hline G1 & 21.1 & 4.4 & 54.0 & 34.3 & 40.4 & 4.1 \\
\hline G2 & 59.3 & 28.7 & 407.3 & 22.0 & 28.5 & 2.6 \\
\hline G3 & 28.9 & 16.6 & 230.6 & 28.2 & 34.7 & 3.5 \\
\hline G4 & 20.0 & 9.0 & 122.0 & 31.6 & 38.1 & 3.8 \\
\hline G5 & 21.7 & 7.4 & 95.3 & 35.4 & 40.3 & 4.0 \\
\hline G6 & 19.4 & 6.3 & 84.0 & 37.0 & 41.7 & 4.1 \\
\hline G7 & 27.8 & 8.6 & 107.7 & 39.4 & 45.6 & 4.5 \\
\hline G8 & 20.0 & 7.6 & 98.9 & 36.0 & 47.2 & 4.7 \\
\hline G9 & 25.0 & 13.3 & 187.8 & 38.3 & 44.2 & 4.4 \\
\hline G10 & 16.1 & 4.4 & 58.1 & 34.0 & 37.7 & 3.7 \\
\hline G11 & 48.3 & 25.0 & 368.7 & 32.2 & 35.6 & 3.3 \\
\hline G12 & 5.00 & 0.4 & 5.1 & 35.8 & 46.2 & 4.0 \\
\hline G13 & 20.0 & 6.2 & 81.7 & 34.2 & 40.5 & 4.0 \\
\hline G14 & 19.4 & 4.7 & 63.7 & 38.7 & 46.0 & 4.6 \\
\hline G15 & 61.0 & 29.0 & 417.3 & 32.8 & 34.2 & 3.0 \\
\hline G16 & 36.7 & 13.2 & 173.8 & 35.6 & 41.1 & 4.1 \\
\hline G17 & 26.7 & 9.9 & 129.7 & 29.7 & 35.3 & 3.5 \\
\hline G18 & 22.8 & 5.9 & 78.8 & 32.4 & 38.2 & 3.8 \\
\hline G19 & 20.0 & 6.4 & 84.1 & 36.0 & 40.1 & 4.0 \\
\hline G20 & 23.9 & 7.9 & 98.4 & 39.7 & 45.6 & 4.5 \\
\hline G21 & 31.7 & 11.9 & 163.3 & 27.7 & 35.2 & 3.5 \\
\hline G22 & 37.2 & 15.4 & 195.7 & 30.6 & 35.3 & 3.5 \\
\hline G23 & 36.1 & 16.3 & 227.1 & 38.2 & 42.7 & 4.3 \\
\hline G24 & 29.4 & 9.8 & 132.6 & 35.9 & 40.8 & 4.0 \\
\hline G25 & 20.0 & 8.6 & 120.6 & 38.1 & 29.4 & 2.9 \\
\hline G26 & 32.8 & 12.7 & 171.1 & 35.9 & 40.7 & 4.0 \\
\hline G27 & 18.9 & 5.3 & 70.7 & 35.2 & 40.2 & 4.0 \\
\hline G28 & 18.9 & 4.9 & 65.7 & 34.3 & 39.8 & 4.0 \\
\hline G29 & 26.1 & 8.9 & 113.8 & 36.5 & 40.9 & 4.1 \\
\hline G30 & 17.8 & 4.3 & 58.4 & 31.7 & 37.0 & 3.7 \\
\hline G31 & 7.8 & 0.95 & 12.7 & 38.5 & 40.5 & 4.1 \\
\hline G32 & 23.3 & 7.3 & 95.5 & 31.5 & 38.0 & 3.8 \\
\hline G33 & 21.1 & 5.0 & 66.5 & 29.6 & 35.7 & 3.6 \\
\hline G34 & 37.2 & 12.5 & 153.2 & 25.4 & 31.1 & 3.1 \\
\hline G35 & 35.6 & 11.6 & 143.3 & 25.0 & 30.9 & 3.1 \\
\hline G36 & 27.8 & 9.4 & 115.0 & 38.2 & 42.8 & 4.3 \\
\hline G37 & 30.6 & 15.5 & 212.3 & 32.0 & 38.3 & 3.8 \\
\hline G38 & 25.6 & 11.8 & 165.6 & 37.6 & 43.4 & 4.3 \\
\hline G39 & 21.7 & 7.2 & 96.9 & 36.2 & 40.0 & 4.0 \\
\hline G40 & 33.9 & 17.0 & 229.8 & 25.1 & 30.0 & 3.0 \\
\hline G41 & 23.9 & 7.2 & 90.9 & 40.7 & 45.6 & 4.5 \\
\hline G42 & 21.7 & 7.5 & 101.9 & 36.6 & 40.1 & 4.0 \\
\hline G43 & 25.6 & 8.7 & 114.3 & 35.8 & 39.7 & 3.9 \\
\hline G44 & 52.2 & 27.0 & 399.3 & 36.4 & 39.7 & 3.7 \\
\hline G45 & 30.0 & 14.7 & 200.3 & 35.1 & 38.6 & 3.8 \\
\hline G46 & 20.0 & 7.5 & 98.2 & 32.0 & 37.7 & 3.8 \\
\hline G47 & 21.7 & 7.7 & 107.3 & 36.7 & 41.7 & 4.2 \\
\hline G48 & 20.6 & 5.7 & 74.4 & 31.3 & 37.0 & 3.7 \\
\hline G49 & 35.6 & 15.0 & 195.4 & 33.7 & 38.3 & 3.8 \\
\hline G50 & 29.4 & 6.4 & 85.1 & 38.8 & 44.7 & 4.5 \\
\hline G51 & 27.8 & 7.4 & 90.1 & 32.9 & 37.8 & 3.8 \\
\hline
\end{tabular}


Table 7. Contd.

\begin{tabular}{lcccccc}
\hline G52 & 8.9 & 1.5 & 19.9 & 38.4 & 47.3 & 4.7 \\
G53 & 28.3 & 9.6 & 120.5 & 33.3 & 38.8 & 3.9 \\
G54 & 23.3 & 7.2 & 85.3 & 38.0 & 42.1 & 4.2 \\
G55 & 20.6 & 7.2 & 95.0 & 39.5 & 43.9 & 4.3 \\
G56 & 21.1 & 4.3 & 55.2 & 32.9 & 38.0 & 3.8 \\
G57 & 20.0 & 5.3 & 74.3 & 41.3 & 46.2 & 4.5 \\
G58 & 41.6 & 25.8 & 376.0 & 35.8 & 42.9 & 3.7 \\
G59 & 62.7 & 33.6 & 430.7 & 32.0 & 36.9 & 3.1 \\
G60 & 6.7 & 0.5 & 6.8 & 37.6 & 43.3 & 4.3 \\
Mean & 26.9 & 10.3 & 139.6 & 34.4 & 39.6 & 3.89 \\
LSD & 7.32 & 3.47 & 43.3 & 2.98 & 4.14 & 0.44 \\
CV & 29.3 & 36.2 & 33.5 & 9.33 & 11.3 & 12.1 \\
\hline
\end{tabular}

${ }^{*},{ }^{* *},{ }^{* * *}$ Significant at $0.05,0.01$ and 0.001 probability levels, respectively; $n s=$ non-significant, SOV $=$ sources of variation, $\mathrm{Df}=$ Degree of freedom, $\mathrm{E}=$ Environment, $\mathrm{G}=\mathrm{Genotype}, \mathrm{GXE}=$ Genotype by Environment Interaction, $\mathrm{FRS}=$ Final rust severity, $\mathrm{Cl}=$ coefficient infection, AUDPC= Area under Disease Progress Curve, $r=$ Apparent infection rate, $D H=$ Days to heading, $D M=$ Days to maturity, $\mathrm{PH}=$ Plant height, $\mathrm{SL}=$ Spike length, NSPS=Number of Spikelet per spike, HLW= Hectoliter weight, TKW = Thousands kernel weight, GY= Grain Yield.

Table 8. Correlation of stem rust disease traits on wheat genotypes.

\begin{tabular}{l|cccc}
\hline & FRS & Cl & AUDPC & r \\
\hline FRS & 1 & & & \\
Cl & $0.894^{* * *}$ & 1 & & \\
AUDPC & $0.877^{* * *}$ & $0.996^{* * *}$ & 1 & \\
$\mathbf{r}$ & $0.447^{* *}$ & 0.330 & 0.324 & 1 \\
\hline
\end{tabular}

*** Significant at 0.001 probability level; $\mathrm{FRS}=$ Final rust severity; $\mathrm{Cl}=$ Coefficient of infection; AUDPC= Area under Disease Progress Curve; $r=$ Apparent infection rate.

the other three stem rust disease measuring traits (FRS, $\mathrm{Cl}$ and AUDPC) with correlation $\left(\mathrm{r}^{2}\right)$ values of $0.447,0.330$ and 0.324 , respectively. This implied that FRS or AUDPC was increasing with the reduction of infection rate over time. i.e. as the epidemic of stem rust disease progressed, only small healthy plant tissue existed for further infections and therefore the rate of epidemic development would be slow. Similar research output was obtained on wheat stem rust resistance studies by Ali et al. (2008). Moreover, a positive and significant correlation of $\mathrm{Cl}$ with AUDPC $\left(\mathrm{r}^{2}=\right.$ 0.996 ) indicated that these two disease traits were related to each other and either of them could be used for selection of stem rust resistant genotypes.

\section{Principal component analysis (PCA)}

The results of principal component analysis designated the contribution of each trait on individual genotypes and thus to the overall genetic variability observed among the tested genotypes. In this study, the first four principal components (PCs) had captured $86.58 \%$ of the total variation among genotypes as indicated in Table 9. PC1 explained $42.61 \%$ of the total variation which mainly was contributed by GY, TKW, CI, AUDPC, and FRS.
The high positive and negative effects of GY, TKW, Cl, AUDPC and FRS on PC1; and the high positive effect of $\mathrm{DH}, \mathrm{DM}$ and $\mathrm{PH}$ on PC2 indicated that these traits are the major contributors to the significant variation among the tested genotypes. Similarly, Alebachew (2012) reported positive and direct attribution of DM and TKW on the grain yield performance of bread wheat genotypes. Mollasadeghi et al. (2011) also ratified that the grain yield of bread wheat genotypes was significantly influenced by number of NSPS, TKW and biological yield of genotypes. Moreover, Nzuve et al. (2013) reported the existence of a highly significant variation $(p<0.001)$ in $F R S, C l$ and AUDPC values of bread wheat genotypes. This therefore indicates that the bread wheat genotypes used in this study were genetically diverse in their stem rust resistance potential and performance of other agronomic traits. Moreover, PC4 showed that the largest share variation was contributed by apparent infection rate of the tested genotypes. This indicated that the redundancy of the trait associated to PC4 and, therefore, the apparent infection rate $(r)$ could be described as redundant descriptor in the description and characterization of the tested genotypes. Similar results were reported by Afutu et al. (2016) on the evaluation of Uganda cowpea germplasm for yield and resistance to scab disease. 
Table 9. Principal components, Eigen values, percentage of total variance, cumulative percentage of variance and eigenvector loadings of twelve traits.

\begin{tabular}{lcccc}
\hline Traits & PC1 & PC2 & PC3 & PC4 \\
\hline FRS & -0.3804 & 0.1599 & 0.2575 & 0.2131 \\
Cl & -0.3728 & 0.1455 & 0.3377 & 0.1344 \\
AUDPC & -0.3666 & 0.1429 & 0.3558 & 0.1212 \\
r & -0.1178 & 0.2759 & -0.1393 & 0.6568 \\
DH & -0.1847 & 0.4627 & -0.1148 & -0.4140 \\
DM & -0.1787 & 0.4615 & -0.1218 & -0.4287 \\
PH & 0.1222 & 0.4325 & -0.3018 & 0.0882 \\
SL & 0.1717 & 0.2771 & -0.4133 & 0.3292 \\
NSPS & 0.3293 & 0.2236 & 0.3316 & 0.0118 \\
HLW & 0.2812 & 0.2262 & 0.3453 & -0.0902 \\
TKW & 0.3499 & 0.2009 & 0.3344 & 0.0634 \\
GY & 0.3844 & 0.1560 & 0.2069 & 0.0829 \\
Eigen values (Explained variance) & 5.11 & 2.83 & 1.49 & 1.04 \\
Percentage of total variance & 42.61 & 23.59 & 12.38 & 8.00 \\
Cumulative percentage of variance & 42.61 & 66.20 & 78.58 & 86.58 \\
\hline
\end{tabular}

Where $\mathrm{FRS}=$ final rust severity, $\mathrm{Cl}=$ Coefficient of infection, $\mathrm{AUDPC}=$ Area under disease progress curve, $r=A p p a r e n t$ infection rate, $\mathrm{DH}=$ Days to heading, $\mathrm{DM}=$ Days to maturity, $\mathrm{PH}=$ Plant height, $\mathrm{SL}=$ Spike length, NSPS=Number of spikelet per spike, HLW= Hectoliter weight, TKW=Thousands kernel weight, $G Y=$ Grain yield.

\section{Cluster analysis (CA)}

The results of cluster analysis which constructed using the mean values of FRS, Cl, AUDPC and $r$ are presented in Figure 1. Bread wheat genotypes that showed the same susceptibility or resistance reaction are presented with the same font color. The genotypes that are displayed in green, orange and red colours were identified as resistant, susceptible and very susceptible genotypes to stem rust disease, respectively. The genotypes with blue and black colours were considered as moderately resistant and moderately susceptible genotypes, respectively. This cluster analysis grouped the tested bread genotypes into five major groups: Cluster-I which accounted for the largest weight consist of 36 moderately stem rust resistant genotypes with AUDPC (54-132.6) and $\mathrm{Cl}$ (4.31-9.94) values. Cluster-II comprised four stem rust resistant genotypes which had small mean values of AUDPC (5.119.9) and $\mathrm{Cl}(0.37-1.48)$. Cluster -IV had 6 moderately stem rust susceptible genotypes with a magnitude of (143.3-173.8) and (11.6-13.19) for AUDPC and $\mathrm{Cl}$, respectively. While Clusters III and V consisted of 8 and 6 genotypes which were susceptible and very susceptible to stem rust disease, respectively. This indicated the existence of genetic divergence in bread wheat genotypes in response to stem rust disease particularly race TTKSK. Earlier studies of Beteselassie et al. (2007) and Ali et al. (2008) reported the existence of genetic variability in stem rust resistance among wheat germplasms. The existed genetic diversity can be exploited during introgression of stem rust resistance genes into susceptible adapted varieties using conventional and genetic engineering approaches.

\section{Conclusions}

This study revealed the existence of significant variations among the tested 60 bread wheat genotypes in terms of their response to Ug99 and performance of their yield and agronomic traits that could be used to selecting parental materials for improving yield and stem rust (Ug99) resistance performance of bread genotypes. Four bread wheat genotypes (G12, G60, G31 and G52) were found to be resistant to Ug99 (race TTKSK) across the three locations and could be used as source of stem rust resistance in future wheat improvement programs.

\section{CONFLICT OF INTEREST}

The authors declare that they have no conflict of interest.

\section{ACKNOWLEDGEMENTS}

Sincere gratitude to DAAD-RUFORUM (Regional Universities Forum for Capacity Building in Agriculture) Scholarship Program for financial support of this study. We would like to also thank the wheat research teams of Debrezeit, Adet and Kulmsa Agricultural Research Centers for providing wheat germplasms, experimental land and helping us during planting and harvesting of the experimental trials. 


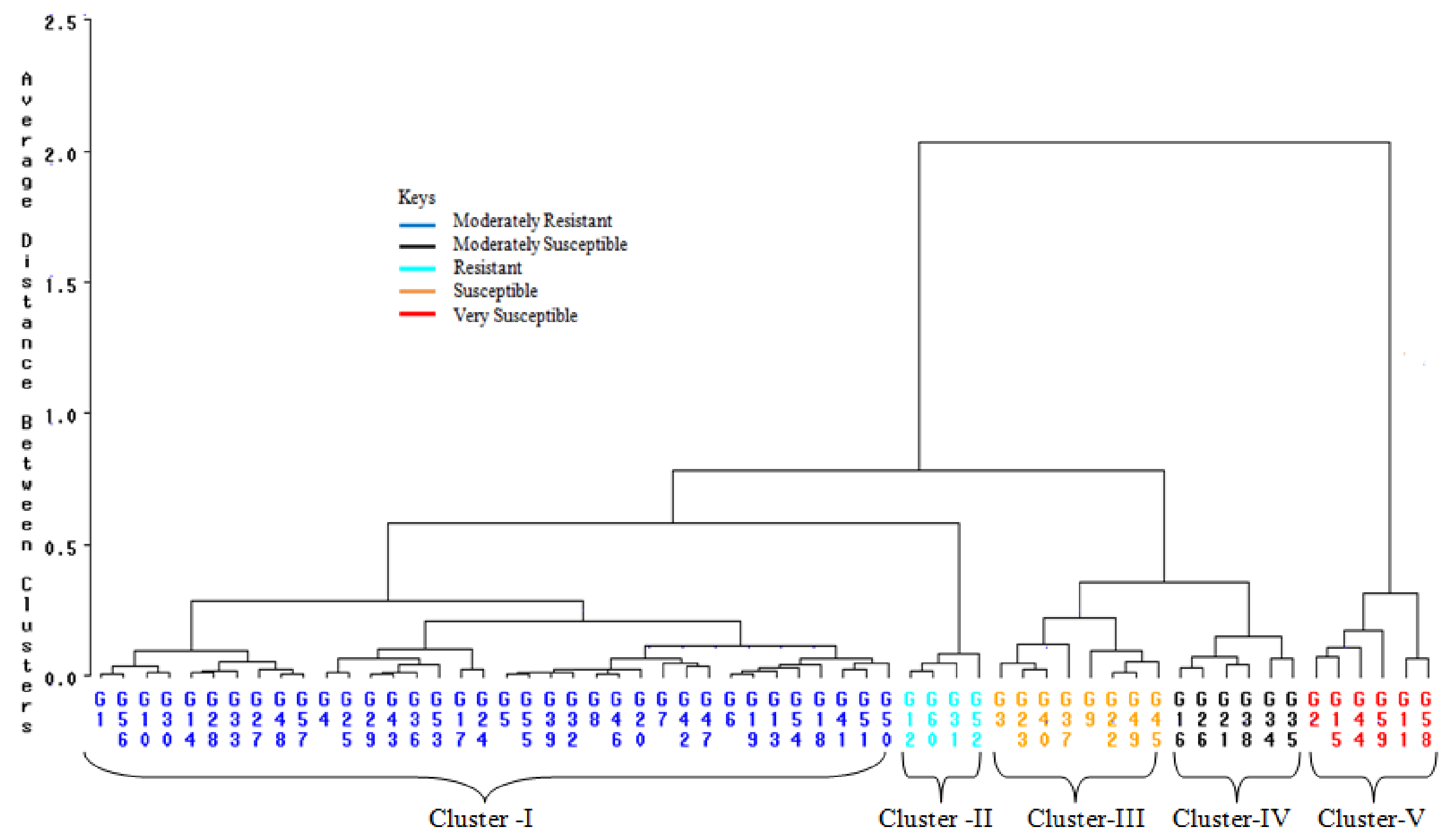

Figure 1. Cluster dendogram of the 60 bread wheat genotypes based on four stem rust disease measuring traits. 


\section{REFRENCES}

Acquaah, G. (2007). Principles of Plant Genetics and Breeding. Blackwell Publishing Limited. Oxford, UK. 569p.

Afutu, E., Mohammed, K. E., Odong, T. L., Biruma, M., \& Rubaihayo, P. R. (2016). Evaluation of Ugandan cowpea germplasm for yield and resistance to scab disease. American Journal of Experimental Agriculture, 12(2), 1-18.

Agrios, G. N. (2005). Plant Pathology, 5th edition. Elsevier Academic Press. Burlington, USA. p. 922.

Alebachew, H. (2012). Participatory and performance evaluation of improved bread wheat (Triticum aestivum L.) varieties in degua tibien and ofla woredas of tigray region, Ethiopia M.Sc. Thesis, Haramaya University, Haramaya, Ethiopia.

Ali, S., Shah, S. J. A., \& Maqbool, K. (2008). Field-based assessment of partial resistance to yellow rust in wheat germplasm. Journal of Agriculture \& Rural Development, 6(1), 99-106.

Beteselassie, N., Fininsa, C., \& Badebo, A. (2007). Sources of stem rust resistance in Ethiopian tetraploid wheat accessions. African journal of Crop Science, 15(1), 51-57.

Denbel, W., Badebo, A., \& Alemu, T. (2013). Evaluation of Ethiopian commercial wheat cultivars for resistance to stem rust of wheat race Ug99. International Journal of Agronomy and Plant Production, 4(1), 15-24.

Draz, I. S., Abou-Elseoud, M., Kamara, A., Alaa-Eldein, O. A., \& ElBebany, A. (2015). Screening of wheat genotypes for leaf rust resistance along with grain yield. Annuals of Agricultural Sciences, 60(1), 29-39.

Duncan, C., Pascal, P., Okwiri, O., Peter, N., Peter, F., \& Sridhar, B. (2015). Evaluation of advanced wheat (Triticum aestivum L.) lines for stem rust (Puccinia graminis f. sp. tritici) resistance and yield. International Journal of Agronomy and Agricultural Research, 6(3), 57-70.

Gamalat, A., \& Elsawi, S. (2015). Inheritance of stem rust resistance and some yield components in crosses from five Egyptian wheat cultivars. Egypt Journal of Plant Breeding, 19(1), 71-87.

Haile, D., Nigussie, D., \& Ayana, A. (2012). Nitrogen use efficiency of bread wheat: Effects of nitrogen rate and time of application, Journal of Soil Science and Plant Nutrition, 12(3), 389-409.

Hailu, E., Woldaeb, G., Danbali, W., Alemu, W and Abebe, T.2015. Distribution of stem rust (Puccinia graminis $f$. sp. tritici) races in Ethiopia. Advances in crop science and technology, 3(3),171-173.

Hair, J. F., Jr Black, W. C., Babin, B. J., \& Anderson, R. E. (2010). Multivariate Data Analysis (7th edition). Pearson Prentice Hall.

Hundie, B., Yirga, F., Kassa, D., Hailu, E., Negash, T., Tesfaye, T., Bacha, N., Shewaye, Y., Woldeab, G., Zegaye, H., \& Tadesse, Z. (2018). Evaluation of Advanced Bread Wheat Lines for Field and Seedling Resistance to Stem Rust (Puccinia graminis f. sp. tritici). American Journal of Biological and Environmental Statistics, 4(2), 74-82.

Jaleta, M., Hodson, D., Abeyo, B., Yirga, C., \& Erenstein, O. (2019). Smallholders' coping mechanisms with wheat rust epidemics: Lessons from Ethiopia. PLoS ONE, 14(7), e0219327.

Kosgey, Z., Owuoche, J. O., Okiror, M. A., \& Njau, P. N. (2015). Inheritance of stem rust (Puccinia graminis Pers.F. Sp. Tritici ericks and $E$. Hen) resistance in bread wheat (Triticum aestivum L.) lines to TTKST race. International Journal of Agronomy and Agricultural Research, 7(4),1-13.

Mclntosh, R. A., Wellings, C. R., \& Park, R. F. (1995). Wheat Rusts: An atlas of resistance genes. Plant Breeding Institute, the University of Sydney, CSIRO Australia.

Mollasadeghi, M. V., Imani A. A., Shahryari, R., \& Khayatnezhad, M. (2011). Correlation and path analysis of morphological triats in different wheat genotypes under end drought stress condition. Middle East Journal of Scientific Research, 7(2), 221-224.
Nzuve, F., Tusiime, G., Bhavani, S., Njau, P., Wanyera, R. (2013). Studies of the genetics of inheritance of stem rust resistance in bread wheat. African Journal of Biotechnology, 12(21), 3153-3159.

Olivera, P. D., Rouse, M., \& Jin, Y. (2018). Identification of new sources of resistance to wheat stem rust in Aegilops spp. in the tertiary gene pool of Wheat. Frontiers in Plant Science, 9, 1719.

Payne, R. W., Murray, D. A., Harding, S. A., Baird, D. H., \& Soutar, D. M. (2013). Genstat for Windows Introduction. 16th Edition, VSN International, Hemel Hempstead.

Peterson, R. F., Campbell, A., \& Hannah, A. E. (1948). A diagrammatic scale for estimating rust intensity on leaves and stems of cereals. Canadian Journal of Research, 26(5), 496-500.

Priyamvada, Saharan, M. S., \& Ratan, T. (2011). Durable resistance in wheat. International Journal of Genetics and Molecular Biology, 3(8), 108-114

Roelfs, A. P., Singh, R. P., \& Saari, E. E. (1992). Rust diseases of wheat: Concepts and methods of disease management. CIMMYT, Mexico, D.F. ISBN: 968-6127-70-4, p. 81.

Safavi, S. A., Ahari, A. B., Afshari, F., \& Arzanlou, M. (2010). Slow rusting resistance in 19 promising wheat lines to yellow rust in Ardabil, Iran. Pakistan Journal of Biological Sciences, 13(5), 240244.

Safavi, S. A., Ahari, A. B., Afshari, F., \& Arzanlou, M. (2013). Slow rusting resistance in Iranian barley cultivars to Puccinia striiformis f. sp. hordei. Journal of Plant Protection Research, 53(1), 5-11.

Sahoo, A., Beyene, L. M., Shiferaw, B., \& Sika, G. (2016). Economywide impacts of technological change in food staples in Ethiopia: A macro-micro approach. MPIA working paper serie 2016-17.

Sikharulidze, Z., Mgeladze, L., Rusudan, D. R, Natsarishvili, K., \& Chkhutiashvili, N. (2015). Reaction of wheat germplasm to stem rust in Georgia. Ekin Journal of Crop Breeding and Genetics, 1(1), 63-68.

Singh, R. P., Hodson, D. P., Huerta-Espino, J., Jin, Y., Bhavani, S., Njau, P., Herrera-Foessel, S., Singh, P.K., Singh, S., \& Govindan, V. (2011). The emergence of Ug99 races of the stem rust fungus is a threat to world wheat production. Annual Review of Phytopathology, 49, 465-481.

Stakman, E. C., Steward, D. M., \& Loegering, W. Q. (1962). Identification of physiological races of Puccinia graminis var. tritici. USDA: Agricultural research service, E-617,1-53.

Stubbs, R. W., Prescott, J. M., Sarrri, E. E., \& Dubinm, H. J. (1986). Cereal disease methodology manual. CIMMYT, El Batan, Mexico.

Tamene, T., Gemechu, K., \& Hussein, M. (2015). Genetic progresses from over three decades of faba bean (Vicia faba L.) breeding in Ethiopia. Australian Journal of Crop Science, 9(1), 41-48.

Van der Plank, J. E. (1963). Plant diseases: Epidemic and control. Academic Press, New York, 349p.

Wilcox son, R. D., Skovmand, B., \& Atif, A. A. (1975). Evaluation of wheat cultivars for the ability to retard development of stem rust. Annals of Applied Biology, 80(3), 275-281.

Zemede, A., Mekbib, F., Assefa, K., \& Bishaw, Z. (2019). Variability in Ethiopian Durum Wheat under Rainfed Environment Subjected to Drought at Anthesis. Ethiopian Journal of Agricultural Sciences, 29(2), 17-29. 